\title{
Dimensional measurement of surfaces and their sampling
}

\section{T C Woo and R Liang}

The number of the discrete samples for the dimensional measurement of machined surfaces and their coordinates is investigated. Counter to intuition, there need not be quadratically more samples than in the case for sampling lines or curves. To justify this novel scheme, accuracy is defined as the discrepancy of a finite point set. Then, from number theory, a particular sequence of numbers is used to compute the sampling coordinates, resulting in a number that is linear in $1 D$, at the same level of accuracy that is achieved by a $2 D$ uniform distribution. Finally, experimental results of the measurement of machined surfaces modeled as random processes are compiled.

coordinate measurement, optical scanning, surface roughness, lowdiscrepancy point sets

While machined surfaces are 2D, topologically, the characteristics of the features can be classified as $0 D, 1 D$ or $2 \mathrm{D}$ according to tolerancing standards ${ }^{1-3}$. A OD (or point) characteristic is a position (of the center of a hole, for example). 1D (or line) characteristics include straightness, circularity, the profile of a line, angularity, perpendicularity, parallelism, runout and concentricity. 2D (or surface) characteristics include flatness, cylindricity, and the profile of a surface. Perhaps because of the predominance of $1 \mathrm{D}$ characteristics, most of the literature on measurement has been on $1 \mathrm{D}$ features ${ }^{4-11}$. This paper calls attention to the fact that $1 \mathrm{D}$ methods may not extend to $2 \mathrm{D}$. A case in point is in the determination of cylindricity. Conventional wisdom suggests the sectioning of a cylinder, hence transforming a $2 \mathrm{D}$ problem into several 1D ones. Suppose that one inquires how the sectioning should be done. (Clearly, different sectionings will yield different results.) Uniform and random sectioning are typically the answers. Suppose that one pushes a step further by asking how many such sections

Department of Industrial and Operations Engineering, University of Michigan, 1205 Beal Avenue, Ann Arbor, MI 48109-2117, USA Paper received: 22 May 1992. Revised: 3 September 1992 should be taken. Those who are analytically inclined may suggest a number such as two (with the reasoning that the axis of a cylinder is a line, and two points that are the derived centers of circles should suffice). Practitioners of metrology, however, may suggest a number greater than two (with statistical significance in mind).

Perhaps equally disturbing, there is no concensus among the suppliers of equipment. In one report, coordinate-measuring machines (CMMs) by five different vendors were benchmarked ${ }^{12}$. The results disagreed by as much as $50 \%$, leading to the implication that good parts may be rejected and bad parts may be in service. This phenomenon was subsequently termed the divergence of methods ${ }^{13}$.

There are three places where 'methods' are invoked in surface measurement: for data sampling, for data fitting, and for data comparison. The second section of this paper reviews these methods, and brings out the source of divergence: distance, and the choice of a metric. The third section clarifies the criteria in sampling as accuracy and time. On the basis of the work by a Fields medalist, accuracy is expressed by a mathematical notion called the discrepancy of a finite set of $N$ points for which a lower bound exists ${ }^{14}$. Time can therefore be quantified in terms of $N$. Then, a sampling strategy based on the Hammersley sequence ${ }^{15}$ is compared with uniform sampling. It shows a remarkable improvement of nearly quadratic reduction in the number of samples, and hence units of time, while maintaining the same level of accuracy. (Loosely speaking, a quadratic reduction of 250000 points yields 500.) Finally, the implementation of the algorithm and the simulation of inspection on machined surfaces are reported on. The results confirm the theoretical prediction. This paper thus contributes to the convergence of sampling methods for surface measurement. It answers the fundamental question as to where the samples should be located on a surface.

\section{DISTANCE AND METRIC}

Distance is the basic element in computational metrology. Consider the familiar Euclidean distance 
between two points $\left(x_{1}, y_{1}, z_{1}\right)$ and $\left(x_{2}, y_{2}, z_{2}\right)$. For each of the coordinates, the difference, e.g. $\left(x_{1}-x_{2}\right)$, is first captured. Then, the differences are squared and summed, and a square root is taken over the sum:

$$
d_{2}=\left[\left(x_{1}-x_{2}\right)^{2}+\left(y_{1}-y_{2}\right)^{2}+\left(z_{1}-z_{2}\right)^{2}\right]^{1 / 2}
$$

The metric applied to Equation 1 is the so-called $L_{2}$ metric, and it has the general form under the $L_{p}$ metric ${ }^{16}$ of

$$
d_{p}=\left[\left|x_{1}-x_{2}\right|^{p}+\left|y_{1}-y_{2}\right|^{p}+\left|z_{1}-z_{2}\right|^{p}\right]^{1 / p}
$$

The absolute value is taken so as to accommodate the odd and even nature of the exponent $p$ with respect to the sign of the differences. When $p \geqslant 1$, one finds a variety of distances, each having its own merit. In particular, when $p=\infty$, Equation 2 becomes a mechanism for selecting the largest of the three components, as supremum, or the maximal element in the set:

$$
d_{\infty}=\sup \left[\left|x_{1}-x_{2}\right|,\left|y_{1}-y_{2}\right|,\left|z_{1}-z_{2}\right|\right]
$$

The transition from Equation 2 to Equation 3 can be understood by assuming that one of the terms, say $\left|y_{1}-y_{2}\right|$, is the largest of the three. Dividing the entire right-hand side of Equation 2 by this term yields

$$
\begin{aligned}
d_{p}= & \left\{\left[\left(\frac{\left|x_{1}-x_{2}\right|}{\left|y_{1}-y_{2}\right|}\right)^{p}+\left(\frac{\left|y_{1}-y_{2}\right|}{\left|y_{1}-y_{2}\right|}\right)^{p}\right.\right. \\
& \left.\left.+\left(\frac{\left|z_{1}-z_{2}\right|}{\left|y_{1}-y_{2}\right|}\right)^{p}\right]\left|y_{1}-y_{2}\right|^{p}\right\}^{1 / p}
\end{aligned}
$$

The first and the third terms of Equation 4 vanish when $p$ becomes very large, hence effectively rendering the supremum as in Equation 3. Figure 1 shows the various $d_{p} \mathrm{~s}$.

The basic idea in data fitting is that of an elevation in dimensionality. Sample points are not only numerous, but also $0 D$. By fitting a curve through points, or a surface through curves, and hence elevating the

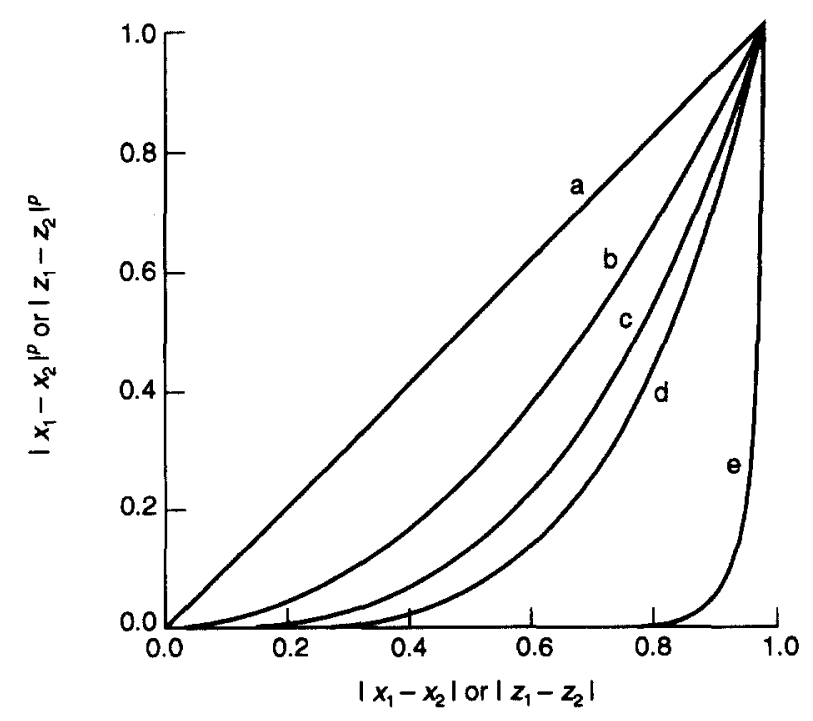

Figure 1. Relative importance of individual parts of Equation 4 for various $\mathrm{p}$

$[\mathrm{a}: p=1, \mathrm{~b}: p=2, \mathrm{c}: p=3, \mathrm{~d}: p=4, \mathrm{e}: p=30$.] dimensionality, two objectives are achieved. First, the size of data storage is reduced, for later algorithmic processing. Second, a group behaviour emerges, for interpretation and decision making. Having seen that distance can adopt infinitely many measures, it should not be surprising that there are a variety of algorithms for elevating the dimensionality of point data by fitting them into curves and surfaces ${ }^{4,6,17}$. The word 'fitting' implies a preconceived notion of some ideal geometry. Indeed, there must be some polynomial of a fixed degree into which the data fits, because, otherwise, fitting becomes arbitrary.

The kinds of ideal geometries in computer-aided design (CAD) are linear, quadratic, cubic and higher-degree. Examples of linear geometry are lines and planes, and examples of quadratic geometry are cylinders, cones and spheres. When the geometry is cubic or higher, there are few familiar shapes, with the possible exception of a torus (which can be used to blend surfaces as fillets). In CAD, these high-degree surfaces are called free-form surfaces. By virtue of the local control that the designer has of the shape, there are various interpolators ${ }^{18}$, such as Hermite, Bernstein and other polynomials, giving rise to Coons, Bézier, B-spline and nonuniform B-spline curves and surfaces that are familiar to the CAD community.

Conceptually, there are three basic criteria to fit data to these ideal geometries: extreme, through and offset. Data can be fitted so that they all lie on the inside (the material side) of the ideal geometry, or, conversely, on the outside, invoking the $\mathrm{L}_{\infty}$ metric. Inscription and circumscription are examples. Fitting an ideal geometry through the data, such that some data lie on the inside and others on the outside, gives a sense of an 'average'. The least-squares method (invoking the $\mathrm{L}_{2}$ metric) is often used. When the mini-max method (invoking the $\mathrm{L}_{\infty}$ metric) is used, the (absolute value of the) maxima imply an offset, providing a connection to a 'tolerance zone'19,20. To ensure that the surrogate geometry satisfies one of the three criteria, a distance function must be invoked, which can be linear, as in Equation 3, or nonlinear, as in Equation 1 or Equation 2. Thus, fitting algorithms are seen as linear or nonlinear optimization problems in general, whereby some form of a (linear or nonlinear) distance is minimized as constrained by an ideal (linear or nonlinear) geometry.

After the data has been fitted, the surrogate geometry is compared with the ideal geometry. Here, again, the notion of distance must be invoked. For speed, one may choose the largest component, as in Equation 3, for an approximation to distance. For accuracy, the RSS distance as in Equation 1 is adopted. Recall that the objective of fitting point data is to elevate the dimensionality. Now that the $0 \mathrm{D}$ points have been raised to $1 \mathrm{D}$ curves or 2D surfaces, the distance between the fitted and the ideal curves and surfaces once again invokes that of the points; to find the distance between two curves, for example, one computes the distance between a pair of closest points on the two curves, and treats it as the distance between the curves. Adopting the paradigm of the $\mathrm{L}_{p}$ metric, one can take the square, the cube and so on as distances. 
Unlike fitting and comparison, the sampling of surfaces has an optimal solution, which is discussed below.

\section{SAMPLING METHOD}

Discrete sampling is inherently an approximation process. If the number of samples is infinite, one may imagine that the error in approximation approaches zero. However, when the sample size is finite, the error must be nonzero. This observation encourages two practical questions. First, for any sampling sequence of size $N$, how small can the error be? This question is answered below. Second, where should the $N$ samples be located? This is addressed in the second section below, in which a surface is assumed to be a unit square addressable by coordinates $(s, t)$, where $s, t \in[0,1]$.

\section{Sampling accuracy}

It has been noted that, once a metric has been adopted, the variety of methods is reduced. However, this still leaves open the following question. Suppose that one adopts the $L_{2}$ metric, what is the best method for sampling? In the last section, the measure of 'goodness' has been subjective: taking the least of the squares or minimizing the maximum. This section reports an objective criterion for assessing sampling errors, and hence the accuracy.

Roth established a lower bound in the discrepancy $D$ for a finite set of $N$ points, in $d$ dimensions ${ }^{14}$ :

$$
D \cong \mathrm{O}\left(N^{-1}\left(\log N^{(d-1) / 2}\right)\right.
$$

The significance of Equation 5 is that the lower bound is expressed only in terms of $N$ and $d$. Most important to dimensional measurement is that one can determine whether a finite set of samples of size $N$, giving rise to a discrepancy $D$, meets Roth's bound, hence giving the least amount of error possible. For the calibration of the sampling sequences, to be discussed later, Roth's bounds in low dimensions are

$$
\begin{array}{ll}
D \cong \mathrm{O}\left((\log N)^{1 / 2} / N\right) & d=2 \\
D \cong \mathrm{O}(1 / N) & d=1
\end{array}
$$

Important as discrepancy is to surface measurement, the concept may be unfamiliar. An outline of the basic idea, with definitions ${ }^{21}$, is given in the Appendix.

\section{Sample size and locations}

The intuition that sample size is inversely proportional to error is confirmed by Equation 7, for 1D. However, as the dimensionality of the sample points increases, the discrepancy is also directly proportional to some power of the logarithm of $N$, as in Equation 6. The key question is that of whether there are points that approach or even reach Roth's bound, as in Equation 5. The answer turns out to be yes.

Before going into sequences, a note on the utility of the samples is required. For a surface under inspection to be visible to an inspection machine, the surface can only be assumed to be single-valued; a command-coordinate pair $\left(x_{i}, y_{i}\right)$ is issued, and a value $z_{i}$ is read. To choose freely between the pairs $\left(x_{i}, y_{i}\right),\left(x_{i}, z_{i}\right)$ and $\left(y_{i}, z_{i}\right)$, the command coordinate $\left(s_{i}, t_{i}\right)$ is identified with the term 'sample', and hence $d=2$. For a point-sampling device such as a CMM, the sequence discussed below can be used directly as command coordinates. For a linesampling device such as a laser scanner, however, data is gathered in arrays. The following sequence can be used as a filter, by postprocessing a given array and selecting those points that are the nearest to the Hammersley coordinates. It is especially the latter, the filtering of optical data, that proves to be powerful, as shown in the last section of this paper.

Sequences have been studied extensively over the years $^{21}$. However, it was van der Corput ${ }^{22}$ who first arrived at a sequence with low discrepancy, using dyadic fractions. Roth ${ }^{14}$ extended the van der Corput sequence to $2 \mathrm{D}$, and Hammersley ${ }^{15}$ to $d$ dimensions. The coordinates $\left(s_{i}, t_{i}\right)$ of a Hammersley point in $2 \mathrm{D}$ are

$$
\begin{aligned}
& s_{i}=i / N \\
& t_{i}=\sum_{j=0}^{k} b_{j} 2^{-j-1}
\end{aligned}
$$

where

$$
\begin{aligned}
i \in & {[0, N-1] } \\
N & =\text { total number of points } \\
k & =\lceil\log i\rceil=\text { ceiling of }(\log i) \\
b_{j} & =\text { binary digits for representing } i
\end{aligned}
$$

As the binary representation of integers is used, the logarithm is taken to the base 2 .

Suppose that $N=10$ Hammersley points are used for sampling. The procedure for computing the Hammersley coordinates is as follows:

- Determine $k$ : Since $i \in[0,9],\lceil\log i\rceil=4$.

- Determine $b_{j}$ : The 4 bit representations $\left(b_{3} b_{2} b_{1} b_{0}\right)$ for $i$ are $\left(\begin{array}{llll}0 & 0 & 0 & 0\end{array}\right),\left(\begin{array}{llll}0 & 0 & 0 & 1\end{array}\right), \ldots,\left(\begin{array}{llll}1 & 0 & 0 & 1\end{array}\right)$.

- Compute $s_{i}$ : According to Equation 8, the $s_{i}$ coordinates for the $N=10$ points are $0 / 10,1 / 10$, $2 / 10, \ldots, 9 / 10$.

- Compute $t_{i}$ : The binary representation for the $t_{i}$ coordinate is obtained by taking the 'mirror image' of the binary representation for $i$ about the decimal point. For instance for $i=1=\left(\begin{array}{llll}0 & 0 & 0 & 1\end{array}\right)$ or $s_{1}=1 / 10$, the mirror image is $\left(\begin{array}{llll}1 & 0 & 0 & 0\end{array}\right)$ or $t_{1}=8 / 16$. For $i=7=\left(\begin{array}{llll}0 & 1 & 1 & 1\end{array}\right)$ or $s_{1}=7 / 10$, the mirror image is (1 11110$)$ or $t_{7}=14 / 16$.

The $N=10$ Hammersley points are shown in Figure 2 . The $10 \times 10$ grid is to be interpreted as the locations for the 100 uniform samples.

The discrepancy of the Hammersley points ${ }^{15}$ in $d$ dimensions is of the order

$$
D_{\mathrm{H}} \cong \mathrm{O}\left(N^{-1}(\log N)^{d-1}\right)
$$

which is off by a factor of one-half power as compared with the absolute lower bound established by Roth in 


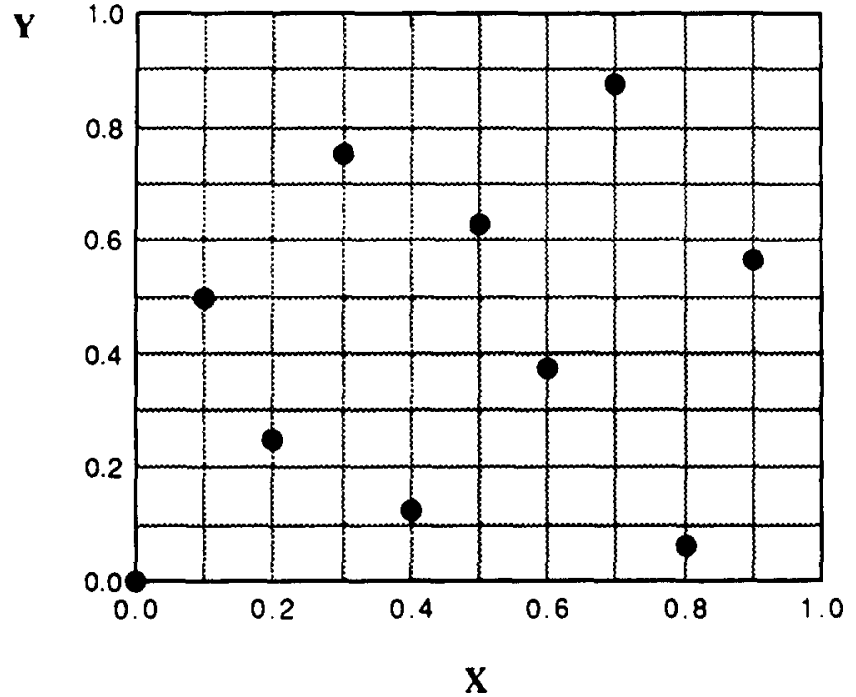

Figure 2. Distribution of ten Hammersley points

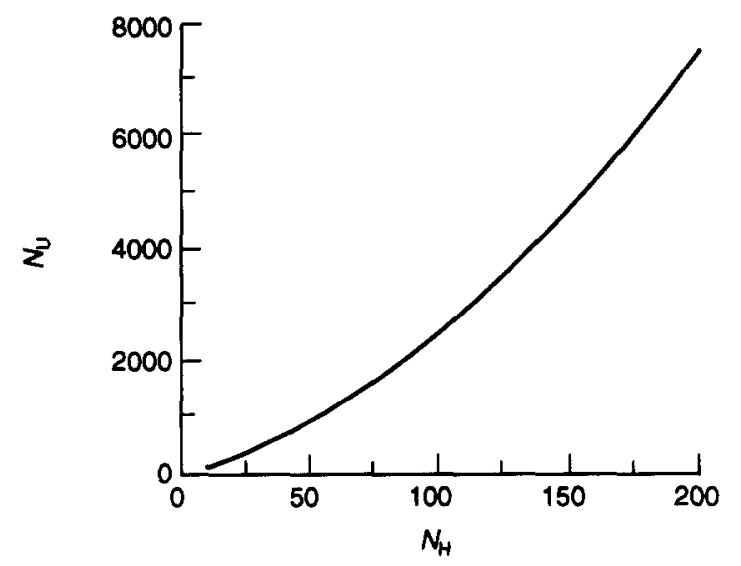

Figure 3. Hammersley points against uniform points, with same accuracy

$\left[N_{\mathrm{U}}=N_{\mathrm{H}}^{2} /\left(\log N_{\mathrm{H}}\right)^{2} \cdot\right]$

Equation 5. While slightly suboptimal, the Hammersley sequence shows a nearly quadratic reduction in the number of points needed by uniform sampling, for the same level of accuracy as measured by discrepancy.

More precisely, it is known from Monte Carlo methods that, as the number of samples $N$ approaches infinity, the error becomes indistinguishable from that of a uniform sequence ${ }^{21}$. In particular, the error for a uniform sequence is $\mathrm{O}\left(N^{-1 / d}\right)$. To compare the number of uniform points $N_{\mathrm{U}}$ against the Hammersley points $N_{\mathrm{H}}$, suppose that they yield the same discrepancy in $2 \mathrm{D}$ :

$$
\frac{1}{N_{\mathrm{U}}^{1 / 2}}=\frac{1}{N_{\mathrm{H}}} \log N_{\mathrm{H}}
$$

It becomes immediately obvious that there are almost quadratically as many $N_{\mathrm{U}}$ as there are $N_{\mathrm{H}}$ :

$$
N_{\mathrm{U}}=\left(\frac{N_{\mathrm{H}}}{\log N_{\mathrm{H}}}\right)^{2}
$$

A graph of the relationship between $N_{\mathrm{U}}$ and $N_{\mathrm{H}}$ is given in Figure 3.

\section{IMPLEMENTATION}

The confluence of mathematics and metrology has many bridges that are yet to be established. There are needs relating to the characterization of errors in the kinematics and dynamics of the measuring machine ${ }^{23}$, the environmental factors such as the temperature gradient ${ }^{24}$, and the manufacturing processes that create the workpiece. Since this paper deals with sampling, it is not desirable to lump all the contributing factors into one. To this end, an 'ideal' measuring machine is assumed, and machined surfaces are simulated as random processes $^{25}$

Not only because random processes offer 1st-order (mean ), 2nd-order (variance) and higher-order statistics ${ }^{26}$ that capture ensemble behaviors of machined surfaces, as evidenced in a beautiful catalog ${ }^{27}$, it turns out that random processes also provide a crucial link of Roth's bound and Hammersley's sequence ${ }^{28}$. In proving the average time complexity for multivariate integration, Wozniakowski ${ }^{29}$ modeled continuous functions as a random process and used the Hammersley sequence for sampling.

Here, machined surfaces are simulated by two models: the Wiener model and the Gaussian model. For each of the two surface models, the uniform and the Hammersley points are used as samples so that their (respective) accuracies can be assessed. Consider the average roughness of a surface $R_{\mathrm{a}}$. This is defined ${ }^{30}$ as the average heights $h$ at a point $(s, t)$ on a surface approximated by $N$ discrete measurements:

$$
R_{\mathrm{a}}=\int_{0}^{1} \int_{0}^{1}|h(s, t)| \mathrm{d} s \mathrm{~d} t \cong \frac{1}{N} \sum_{i=1}^{N}\left|h\left(\mathbf{p}_{s i}, \mathbf{p}_{t i}\right)\right|
$$

where $\mathbf{p}_{s i}$ is the $s$ coordinate of the $i$ th point in a sequence of $N$ points. (The absolute value is taken on $h(s, t)$ in Equation 12 so as to ensure that the asperities do not cancel out.) Calling the difference between the two sides of Equation 12 the error,

$$
\varepsilon\left(N, \mathbf{p}_{i}\right)=\int_{0}^{1} \int_{0}^{1}|h(s, t)| \mathrm{d} s \mathrm{~d} t-\frac{1}{N} \sum_{i=1}^{N}\left|h\left(\mathbf{p}_{i s}, \mathbf{p}_{i t}\right)\right|
$$

The root-mean-square error (RMS error) in surface measurement $\varepsilon_{\mathrm{RMS}}$, by invoking the $\mathrm{L}_{2}$ metric, is simply

$$
\varepsilon_{\text {RMS }}=\left\{\int_{0}^{1} \int_{0}^{1}\left[\varepsilon\left(N, \mathbf{p}_{i}\right)^{2} \mathrm{f}(s, t) \mathrm{d} s \mathrm{~d} t\right\}^{1 / 2}\right.
$$

where $f(s, t)$ is the probability density function for the Wiener or the Gaussian distribution. The sample points $\mathbf{p}_{i}$ in Equation 14 are not specified. However, it is desirable to use a set of points having an $\varepsilon_{\mathrm{RMS}}$ which is as small as possible. Here is where the Hammersley points come in, associated with which is the notion of discrepancy. To use the Hammersley points for sampli $*$ ?. the discrepancy of a point set and the RMS error $1_{11}$ measuring surface roughness must be established. This is done in Reference 31. 
Figure 4 shows 32 Hammersley points superimposed on a Wiener surface and on a Gaussian surface. It may be seen that the Wiener surface is smooth and periodic, simulating single-point rotary cutting. The Gaussian surface is more textured and independent, simulating such processes as electrodischarge machining or sand blasting ${ }^{27}$.

A number of experiments have been conducted to assess the accuracy of the Hammersley points. The number of samples ranges from 16 to 15625 , for two reasons. First, the uniform distribution of $N_{\mathrm{U}}=10 \times 10$, $25 \times 25,50 \times 50$ and $125 \times 125=15625$ points are used as a benchmark. Second, the Hammersley points are chosen at $N_{\mathrm{H}}=16,32,64$ and 128 to approximate the nearly quadratic reduction in Equation 11.20 runs are made for each of the Wiener and the Gaussian surface models, and the RMS error using the Hammersley points versus the RMS error using the uniform points are

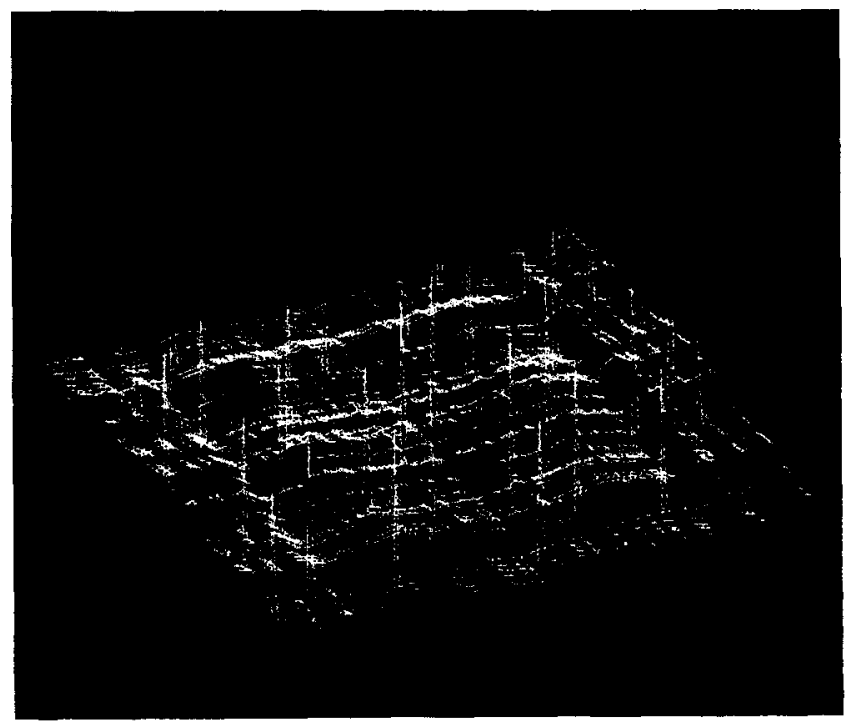

a

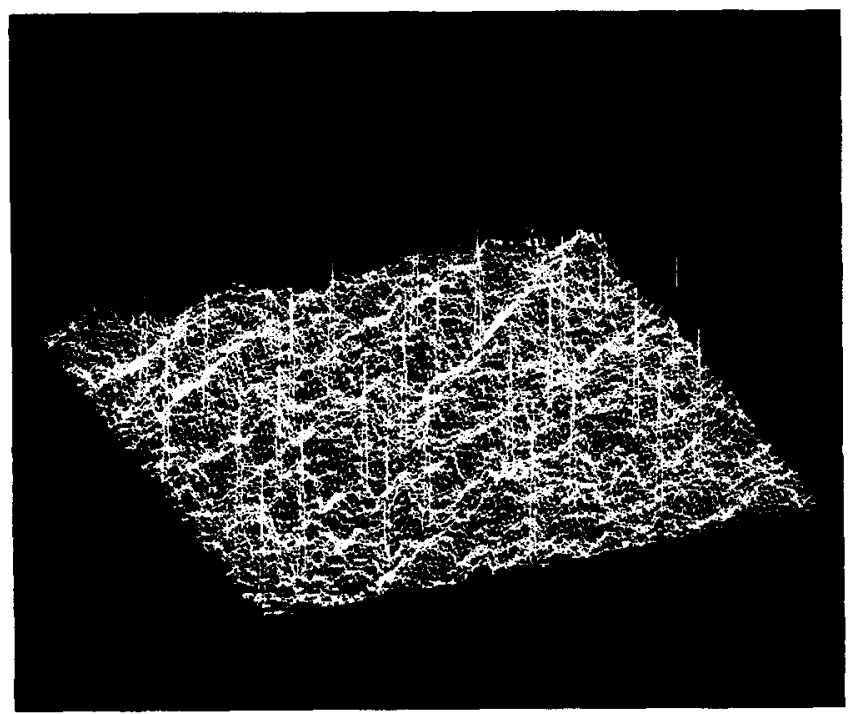

b

Figure 4. Hammersley points on Wiener and Gaussian surfaces; (a) 32 Hammersley points on modified Wiener surface, (b) 32 Hammersley points on Gaussian surface
Table 1. RMS error on Wiener surfaces

\begin{tabular}{lccc}
\hline & \multicolumn{2}{c}{ Hammersley } \\
\hline Number & RMS error & Number & RMS error \\
\hline $50 \times 50$ & 0.01414 & 64 & 0.00678 \\
$25 \times 25$ & 0.03295 & 32 & 0.01081 \\
$10 \times 10$ & 0.09469 & 16 & 0.01407 \\
\hline
\end{tabular}

Table 2. RMS error on Gaussian surfaces

\begin{tabular}{cccc}
\hline \multicolumn{2}{c}{ Uniform } & \multicolumn{2}{c}{ Hammersley } \\
\hline Number & RMS error & Number & RMS error \\
\hline $125 \times 125$ & 0.02254 & 128 & 0.01766 \\
$50 \times 50$ & 0.08092 & 64 & 0.04398 \\
$25 \times 25$ & 0.06107 & 32 & 0.05453 \\
\hline
\end{tabular}

calculated on the basis of Equation 14. Table 1 shows the experimental results for the RMS errors for the Wiener surfaces, with correspondences in the reduced number of sample points.

For an approximately quadratic reduction in the number of points, the Hammersley samples are more accurate than uniform sampling. Results for the 20 Gaussian surfaces are given in Table 2. For an approximately quadratic reduction in the number, the Hammersley points are of the same order of accuracy as the uniform points.

\section{CONCLUSIONS}

This paper has introduced the application of the Hammersley sequence for surface measurement, hence bringing some convergence to sampling methods. The basis for the application of the Hammersley sequence is that it exhibits a discrepancy that approaches the number-theoretic limit asserted by Roth. Discrepancy is related to the RMS error in measuring average surface roughness.

For point sampling, users of the CMM may wish to examine the reported strategy for random or uniform sampling. For line sampling, practitioners in computer vision can note that a scan of $500 \times 500$ points can now be dramatically compacted to approximately its square root.

\section{ACKNOWLEDGEMENTS}

The authors are grateful to the reviewers of this paper, whose insightful comments were most helpful.

This work was supported in part by the Fulbright Foundation, grants DMC-8903029 and DMC-9113791, from the US National Science Foundation, and a grant from the University Research Fund, Scientific Research Laboratory, Ford Motor Company.

\section{REFERENCES}

1 'Engineering drawing practice' BS GS308 British Standards Institute, UK (1972) 
2 'Dimensioning and tolerancing' ANSI Y14.5M American Society of Mechanical Engineers, USA (1982)

3 'Technical drawings - Tolerances of form and of position' ISO Rl101 International Standards Organisation, Switzerland (1982)

4 Cox, M G 'The least squares solution of overdetermined linear equations having band or augmented band structure' IMA J. Numer. Anal. Vol 1 (1981) pp 3-22

5 Chetwynd 'Linearized exchange algorithms in metrology' Proc. Conf. Software for Co-ordinate Measuring Machines National Physical Laboratory, UK (25 Sep 1985) pp 24-29

6 Anthony, G T and Cox, M G 'Reliable algorithms for roundness assessment according to BS3730' Proc. Conf. Software for Co-ordinate Measuring Machines National Physical Laboratory, UK (25 Sep 1985) pp $30-37$

7 Shunmugan, M S New approach for evaluating form error of engineering surfaces' Comput.-Aided Des. Vol 19 No 7 (1987) pp 368-374

8 Le, V $B$ and Lee, D T 'Out-of-roundness problem revisited'IEEE Trans. Pattern Anal. \& Mach. Intell. Vol 13 No 3 (1991) pp 217-223

9 Shunmugan, $\mathbf{M} \mathbf{S}$ 'Criteria for computer-aided form evaluation' Trans. ASME J. Eng. Indust. Vol 113 (1991) pp 233-238

10 Caskey, G, Hari, Y, Hocken, R, Palanvelu, D, Reja, $\mathbf{J}$, Wilson, $\mathbf{R}$, Chen, $\mathbf{K}$ and Yang, $\mathbf{J}$ 'Sampling techniques for coordinate measuring machines' Proc. NSF Design \& Manufacturing Systems Conf. Atlanta, GA, USA (1992) pp 779-786

11 Roy, $U$ and Zhang, $X$ 'Establishment of a pair of concentric circles with the minimum radial separation for assessing roundness error' Comput.-Aided Des. Vol 24 No 3 (1992) pp 161-168

12 Walker, R GIDEP Alert XI-A-88-01 (22 Aug 1988)

13 Schrieber, $\mathbf{R}$ 'The methods divergence dilemma' Manuf. Eng. (1990)

14 Roth, K F 'On irregularity of distribution' Mathematika Vol 1 (1954) pp 73-79

15 Hammersley, $\mathbf{J} \mathbf{M}$ 'Monte Carlo methods for solving multivariate problems' Ann. New York Acad. Sci. Vol 86 (1960) pp 844-874

16 Schreider, Y A What is Distance? University of Chicago Press (1974)

17 Lancaster, P and Salkauskas, K Curve and Surface Fitting Academic Press (1986)

18 Farin, G Curves and Surfaces for Computer Aided Geometric Design Academic Press (1988)
19 Roy, U, Liu, C R and Woo, T C Review of dimensioning and tolerancing: representation and processing' Comput.-Aided Des. Vol 23 No 7 (1991) pp $466-483$

20 Juster, $\mathbf{N} \mathbf{P}$ 'Modelling and representation of dimensions and tolerances: a survey' Comput.-Aided Des. Vol 24 No 1 (1992) pp 3-17

21 Kuipers, $\mathbf{L}$ and Niederreiter, $\mathbf{H}$ Uniform Distribution of Sequences John Wiley, USA (1974)

22 van der Corput, J G 'Verteilungsfunktionen I, II' Nederl. Akad. Wetnesch. Proc. Vol 38 (1935) pp 813-821 and pp 1058-1066

23 Soons, J A, Theuws, F $\mathrm{C}$ and Schellekens, $\mathrm{P}$ H "Modeling the errors of multi-axis machines: a general methodology' Precision Eng. Vol 14 No 1 (1992) pp 5-19

24 Lingard, P S, Purss, M E, Sona, C M, Thwaite, E G and Morisson, $\mathbf{G} \mathbf{H}$ 'Temperature perturbation effects in a high temperature CMM' Precision Eng. Vol 13 No 1 (1991) pp 41-51

25 Peklenik, $\mathbf{J}$ 'Contribution to the theory of surface characterization' Ann. CIRP Vol 12 (1963) pp 173-176

26 Ross, S Stochastic Processes John Wiley (1983)

27 Stout, K J, Davis, $\mathbf{E} \mathbf{J}$ and Sullivan, $\mathbf{P} \mathbf{J}$ Atlas of Machined Surfaces Chapman \& Hall, UK (1990)

28 Cipra, B A 'The breaking of a mathematical curse' AAAS Sci. (1991) p 165

29 Wozniakowski, H 'Average case complexity of multivariate integration' Bull. $A M S$ Vol 24 No 1 (1991) pp 185-194

30 Thomas, T R Rough Surfaces Longman, UK (1982)

31 Liang, $\mathbf{R}$ and Woo, $\mathbf{T} \mathbf{C}$ 'Accuracy and time in measuring surface roughness. Part 1: Mathematical foundations' Technical Report 92-17 Dep. Industrial and Operations Engineering, University of Michigan, USA (1992)

32 Roth, K F 'On irregularity of distribution' Acta Arithmetica Vol 37 (1980) pp 76-75

\section{APPENDIX}

\section{Discrepancy}

In an effort to estimate surface parameters by using a discrete sample of points, it is convenient to begin in $2 \mathrm{D}$. Suppose that there is a rectangular region with sides $t_{1}$ and $t_{2}$, where $0 \leqslant t_{1} \leqslant 1,0 \leqslant t_{2} \leqslant 1$, and one wishes to estimate the area. One estimator is to sample in the unit square at points $\mathbf{p}_{1}, \mathbf{p}_{2}, \ldots, \mathbf{p}_{N}$, where $\mathbf{p}_{i}=\left(s_{i}, t_{i}\right)$ and 
$i \in[1, N]$, and count the number of points that are in

$$
\text { area }=t_{1} * t_{2} \simeq \frac{1}{N} \sum_{i=1}^{N} \chi\left(\mathbf{p}_{i}\right)
$$

Here, $\chi\left(\mathbf{p}_{i}\right)$ is the characteristic function indicating whether or not a point $\mathbf{p}_{i}$ is in the region:

$$
\chi\left(\mathbf{p}_{i}\right)= \begin{cases}1 & \left(\mathbf{p}_{i}\right) \text { is in the region } \\ 0 & \text { otherwise }\end{cases}
$$

The error, called the residual, is

$$
\text { residual }=t_{1} * t_{2}-\frac{1}{N} \sum_{i=1}^{N} \chi\left(\mathbf{p}_{i}\right)
$$

Generalizing Equation 17 to $d$ dimensions, whereby the unit square becomes the unit hypercube $I^{d}=[0,1]^{d}$ parameterized by $\mathbf{t}$, the residual becomes

$$
R\left(\mathbf{t}, N, \mathbf{p}_{i}\right)=\prod_{k=1}^{d} t_{k}-\frac{1}{N} \sum_{i=1}^{N} \chi\left(\mathbf{p}_{i}\right)
$$

The discrepancy in the $\mathrm{L}_{2}$ norm, or $L_{2}$ discrepancy, is defined as ${ }^{21}$

$$
D_{2}=\left\{\int_{I^{d}}\left[R\left(\mathbf{t}, N, \mathbf{p}_{i}\right)\right]^{2} \mathrm{~d} \mathbf{t}\right\}^{1 / 2}
$$

Roth $^{14,32}$ established the lower bound on the $L_{2}$ discrepancy as

$$
D_{2} \geqslant k N^{-1}(\log N)^{(d-1) / 2}
$$

where the constant $k$ is in terms of the dimension $d$ only. 